\title{
Generalised states: a multi-sorted algebraic approach to probability
}

\author{
Tomáš Kroupa • Vincenzo Marra \\ This paper is dedicated to the memory of Franco Montagna.
}

Received: date / Accepted: date

\begin{abstract}
We introduce a generalised notion of state as an additive map from a Boolean algebra of events to an arbitrary MV-algebra. Generalised states become unary operations in two-sorted algebraic structures that we call state algebras. Since these, as we show, form an equationally defined class of algebras, universal-algebraic techniques apply. We discuss free state algebras, their geometric representation, and their connection with the theory of affine representations of lattice-groups.
\end{abstract}

Keywords probability - state $\cdot$ multi-sorted algebra heterogeneous algebra · Boolean algebra · MV-algebra . Lattice-ordered Abelian group.

\section{Introduction}

Classically, states are normalised positive linear functionals on Riesz spaces with unit, or more generally, on lattice-ordered Abelian groups with unit. States provide the standard abstraction of the expected value operator. States of MV-algebras [14] are just the same thing as classical states, up to translation through the wellknown theory of the functor $\Gamma[4$, Chapter 7]. However, because of the relation between MV-algebras and Boolean algebras, MV-algebraic states may also be regarded as a many-valued generalisation of finitely additive probability assignments. Here, families of (manyvalued) events are no longer modelled by Boolean algebras, but rather by MV-algebras. MV-algebraic states

The work of T. Kroupa was supported by Marie Curie IntraEuropean Fellowship OASIG (PIEF-GA-2013-622645).

T. Kroupa · V. Marra

Dipartimento di Matematica "Federigo Enriques",

Università degli Studi di Milano,

Via Cesare Saldini 50, 20133 Milano, Italy

E-mail: \{tomas.kroupa,vincenzo.marra\}@unimi.it have been intensively investigated in the last two decades; see [6] for a recent survey of results. On MValgebraic matters we follow the notation and terminology of [4], to which we refer the reader for background. Given an MV-algebra $(D, \oplus, \neg, 0)$, we introduce as usual the binary operations $\odot, \ominus$, and the constant 1 together with the lattice supremum $\vee$ and infimum $\wedge$ on $D$. The lattice order of $D$ is denoted by $\leq$.

In 2009, Flaminio and Montagna [7] introduced the notion of internal state as an additional unary operation $s: D \rightarrow D$ on an MV-algebra $D$. Internal states are intended to capture the basic properties of states in a setting amenable to universal-algebraic techniques. Indeed, in [7, Definition 3.1], $s$ is required to satisfy certain equational laws. (See Remark 1 below for the Flaminio-Montagna axiomatisation.) Please see the references in [6, Section 8] for subsequent work on internal states. On the other hand, a state of an $M V$-algebra $D$ is a mapping $s: D \rightarrow[0,1]$ satisfying $s(1)=1$ and the following condition for every $a, b \in D$ :

if $a \odot b=0$, then $s(a \oplus b)=s(a)+s(b)$.

Thus:

- a state is defined on an MV-algebra and takes values in the real unit interval, and

- the form of the key axiom (1) is not equational.

Prima facie, therefore, there seems to be no hope of directly applying the theory of universal algebra to states. One way to remedy this is to make recourse to the Flaminio-Montagna theory of internal states. Unfortunately, that comes at the significant cost of renouncing the fundamental conceptual distinction between events - elements of the domain of a state - and degrees of probability - elements of the co-domain of the state. 
The purpose of this note is to show that, pace first impressions, an equational theory of states that preserves this conceptual distinction is indeed feasible; more substantially, we also provide sufficient mathematical evidence to indicate that its developments may prove of considerable interest. Specifically, we provide a preliminary representation theory for free objects in the resulting equationally definable class of algebras. Our main point can already be made in the classical setting of Boolean algebras of events, and we shall therefore work in that setting. We do emphasise that it is straightforward to adapt our definitions to MV-algebras of manyvalued events; compare Remark 1 . We further alert the reader to the fact that $\mathrm{MV}$-algebras already play a key rôle in this classical setting, as abstractions of the codomain $[0,1]$ of finitely additive probabilities.

Throughout this note we think of a Boolean algebra $E$ as an abstract algebraic model of the structure of events in probability theory, an event being an element of $E$. The underlying order relation on a Boolean algebra is written $\subseteq$, and the binary relative complement operation is written $\backslash$. We stress for clarity that our definitions need no lattice-theoretic completeness assumption about $E$. As is well known, Boolean algebras are precisely those MV-algebras such that $\oplus$ is idempotent, and then $\oplus$ agrees with join and $\odot$ agrees with meet. Because we shall soon have to work with Boolean algebras and MV-algebras within a single formal framework, we need to distinguish the Boolean operations from the MV-algebraic ones. Hence we write

$$
\left(E, \cup, \cap,^{\prime}, \perp, \top\right)
$$

to denote a Boolean algebra with its operations and their obvious meaning. Now, if $D=E$ is a Boolean algebra, states of $E$ clearly coincide with finitely additive probability assignments of $E$. More precisely, a state of a Boolean algebra $E$ is a mapping $s: E \rightarrow[0,1]$ satisfying $s(\top)=1$ and the following condition for every $a, b \in E$ :

if $a \cap b=\perp$, then $s(a \cup b)=s(a)+s(b)$.

Terminology. For the rest of this note, state means state of a Boolean algebra.

If Boolean algebras are algebraic models of families of events, what is an abstract model of the set $[0,1]$ of probability degrees? In the remainder of this note, we make the following points.

(I) The relevant structure of $[0,1]$ as the collection of probability degrees includes its MV-algebraic structure.
(II) In light of (I), one can replace $[0,1]$ by any MValgebra in the definition of state (2), thereby obtaining what we call generalised states (see Definition 1).

(III) Furthermore, generalised states admit an equational definition - hence, a fortiori, so do states.

(IV) Items (I-III) show that generalised states, when properly interpreted, can be regarded as genuine algebraic structures, provided only one allows multisorted algebras. (We comment on multi-sorted universal algebra in Section 4.)

(V) Item (IV) thus leads to the notion of state algebra, a two-sorted algebraic structure equipped with a generalised state as a multi-sorted operation. In closing, we discuss free state algebras and prove a significant representation theorem for a class of them.

\section{Generalised states}

The basic operations required in elementary probability theory on the probability degrees themselves - not on the events - are the following.

(i) Addition of real numbers.

(ii) Involution in $[0,1]$, that is, $x \in[0,1] \mapsto 1-x \in[0,1]$.

(iii) Infima and suprema derived from the natural order of the real numbers.

Now, (i-iii) amount to considering $(\mathbb{R}, 1)$ as a latticeordered Abelian group with unit 1 . It may be argued that one should add to (i-iii) the multiplicative structure of $[0,1]$, for example to model independence of events, or conditioning. However, for the purposes of this note we do not consider multiplication.

The addition in (i) can only be restricted to $[0,1]$ at the price of turning it into a partially defined operation. However, by the theory of the functor $\Gamma$ [4, Chapter 7], the unital lattice-group $(\mathbb{R}, 1)[1]$ is uniquely determined by the MV-algebra $[0,1]$, its unit interval. Hence, in place of (i-ii) we can equivalently consider the MValgebraic structure of $[0,1]$. The order in (iii) is then derivable from the MV-algebraic structure in the usual manner.

In light of the above, let $(G, u)$ be any lattice-ordered Abelian group with strong order unit $u$, and let $E$ be any Boolean algebra. We can define a generalised notion of $(G, u)$-valued state by considering functions

\section{$s: E \rightarrow G$}

that satisfy the normalisation condition $s(\top)=u$ and the finite additivity condition

if $a \cap b=\perp$, then $s(a \cup b)=s(a)+s(b)$. 
Passing to the unit-interval MV-algebra $\Gamma(G, u)=[0, u]$, whose truncated addition is $x \oplus y:=(x+y) \wedge u$ for all $x, y \in[0, u]$, such functions turn out to be in one-one correspondence with the functions $s: E \rightarrow[0, u]$ that satisfy

$s(\top)=u$, and

if $a \cap b=\perp$, then $s(a \cup b)=s(a)+s(b)$.

We will show in Lemma 1 below how to turn the quasiequational notion (3-4) into an equivalent equational one, thus achieving item (III) in the Introduction.

\section{Definition 1 (Generalised state)}

A generalised state of a Boolean algebra $E$, with values in an MV-algebra $D$, is a mapping $s: E \rightarrow D$ such that for every $a, b \in E$ the following hold.

(A1) $s(a \cup b)=s(a) \oplus s(b \backslash a)$,

(A2) $s\left(a^{\prime}\right)=\neg s(a)$,

(A3) $s(T)=1$.

Remark 1 Our Definition 1 is inspired by but not identical to the one used in [7]. If the domain of $s$ were an MV-algebra rather than a Boolean algebra, then condition (A1) would need to be replaced by

$\left(\mathrm{A}^{\prime}\right) \quad s(a \oplus b)=s(a) \oplus s(b \ominus(a \odot b))$

in order to prove an analogue of our Lemma 1 below. Indeed, Flaminio and Montagna defined an internal state of the MV-algebra $D$ to be a map $s: D \rightarrow D$ that satisfies $\left(\mathrm{A} 1^{\prime}\right),(\mathrm{A} 2)$, and (A3), together with the additional axiom

(A4) $s(s(a) \oplus s(b))=s(a) \oplus s(b)$

expressing the fact that the image of $D$ under $s$ is an MV-subalgebra of $D$. Further, other authors introduced and studied a concept of state for residuated lattices, allowing for values in an arbitrary residuated lattice a generalised state in the sense of our Definition 1 is called, in that literature, an "order-preserving type I state"; see [5, Proposition 3.22].

Notation. For the rest of this note, $E$ always denotes a Boolean algebra, $D$ always denotes an MV-algebra, and $s$ always denotes a function $s: E \rightarrow D$.

Let us collect useful elementary facts about generalised states in

Proposition 1 If $s$ is a generalised state, the following hold for every $a, b \in E$.

1. $s(\perp)=0$.

2. If $a \subseteq b$, then $s(a) \leq s(b)$.

3. $s(a \cup b) \leq s(a) \oplus s(b)$, and if $a \cap b=\perp$, then $s(a \cup b)=$ $s(a) \oplus s(b)$.
4. $s(a \backslash b) \geq s(a) \ominus s(b)$, and if $b \subseteq a$, then $s(a \backslash b)=$ $s(a) \ominus s(b)$.

5. $s(a \cap b) \geq s(a) \odot s(b)$, and if $a \cap b=\perp$, then $s(a) \odot$ $s(b)=0$.

6. $s(a \cup b)=s(a) \oplus[s(b) \ominus s(a \cap b)]$.

Proof 1. By (A2) and (A3), $s(\perp)=\neg s(\top)=0$.

2. Let $a \subseteq b$. Then $b=a \cup(b \backslash a)$. Since $a \cap(b \backslash a)=\perp$, we get

$s(b)=s(a \cup(b \backslash a))=s(a) \oplus s(b \backslash a) \geq s(a)$.

3. It follows from (A1) and item 2 that

$s(a \cup b)=s(a) \oplus s(b \backslash(a \cap b)) \leq s(a) \oplus s(b)$.

Let $a \cap b=\perp$. Then

$s(a \cup b)=s(a) \oplus s(b \backslash \perp)=s(a) \oplus s(b)$.

4. We get

$$
\begin{aligned}
s(a \backslash b) & =s\left(\left(a^{\prime} \cup b\right)^{\prime}\right)=\neg s\left(a^{\prime} \cup b\right) \\
& \geq \neg(\neg s(a) \oplus s(b))=s(a) \odot \neg s(b)=s(a) \ominus s(b) .
\end{aligned}
$$

Let $b \subseteq a$. Then $a^{\prime} \cap b=0$ and the equality in the formula above follows from item 3 .

5. We get

$$
\begin{aligned}
s(a \cap b) & =s\left(a \backslash b^{\prime}\right) \\
& \geq s(a) \ominus s\left(b^{\prime}\right)=s(a) \odot \neg s\left(b^{\prime}\right)=s(a) \odot s(b) .
\end{aligned}
$$

Let $a \cap b=\perp$. Then

$s(a) \odot s(b) \leq s(a \cap b)=s(\perp)=0$.

6. This is a consequence of (A1) and item 4.

We can now prove:

Lemma 1 Let $s: E \rightarrow D$ satisfy $s(\top)=1$. Then the following are equivalent.

1. $s$ is a generalised state.

2. For every $a, b \in E$, if $a \cap b=\perp$, then $s(a \cup b)=$ $s(a) \oplus s(b)$ and $s(a) \odot s(b)=0$.

3. For every $a, b \in E$, if $a \cap b=\perp$, then $s(a \cup b)=$ $s(a)+s(b)$, where + is the addition operation of the essentially unique lattice-ordered Abelian group $(G, 1)$ such that $\Gamma(G, 1)=D$.

Proof The implication from item 1 to 2 follows from Proposition 1. Conversely, let a mapping $s: E \rightarrow D$ satisfy $s(\top)=1$ and 2 . Then $a \cap a^{\prime}=0$ yields $s(a) \odot$ $s\left(a^{\prime}\right)=0$ and

$1=s(\top)=s\left(a \cup a^{\prime}\right)=s(a) \oplus s\left(a^{\prime}\right), \quad a \in E$.

Therefore $s\left(a^{\prime}\right)=\neg s(a)$ by [4, Lemma 1.1.3] and thus (A2) in Definition 1 holds. Finally, we prove (A1). Using 
the identities $a \cap(b \backslash a)=\perp, a \cup b=a \cup(b \backslash a)$, we obtain

$s(a \cup b)=s(a \cup(b \backslash a))=s(a) \oplus s(b \backslash a), \quad a, b \in E$.

Hence, (A1) in Definition 1 holds.

It remains to show that item 2 is equivalent to item 3. This amounts to the well-known fact that in any MValgebra $x \oplus y$ agrees with $x+y$ of its enveloping unital Abelian lattice-group if, and only if, $x \odot y=0$ - see [4, Lemma 2.1.3(i)].

As promised, Lemma 1 shows that a generalised state can be thought of as an additive map $E \rightarrow D$, that is to say, it is the same thing as a function satisfying (3-4).

Using Proposition 1 we can easily derive the following inequalities, which can be viewed as analogues of the well-known Boole-Fréchet bounds in probability theory, see e.g. $[9, \S 3.5]$.

Corollary 1 (Boole-Fréchet bounds) If $s$ is a generalised state then the following hold for all $a, b \in E$.

$s(a) \vee s(b) \leq s(a \cup b) \leq s(a) \oplus s(b)$

$s(a) \odot s(b) \leq s(a \cap b) \leq s(a) \wedge s(b)$

Further, these bounds are attained for appropriate choices of $D, E, s, a$, and $b$.

Proof We use Proposition 1. Both $s(a) \vee s(b) \leq s(a \cup b)$ and $s(a \cap b) \leq s(a) \wedge s(b)$ follow from monotonicity (item 2). The inequalities $s(a \cup b) \leq s(a) \oplus s(b)$ and $s(a) \odot s(b) \leq s(a \cap b)$ are item 3 and 5 , respectively. To show the bounds are attained, it suffices to take $D=E$ and $s$ the identity homomorphism, which clearly is a generalised state.

Remark 2 The notion of observable introduced by Pulmannová in [17] is formally the same as our concept of generalised state. Indeed, an observable was defined in that paper as a mapping $x$ from a Boolean algebra $E$ into an MV-algebra $D$ satisfying $x(\top)=1$ and condition 2 in Lemma 1. The motivation for studying observables in [17] comes from quantum probability theory.

\section{Examples of generalised states}

We discuss in this section two examples of generalised states that are significant for different reasons. We first point out a few easy facts.

Clearly, any state of a Boolean algebra $E$ is a generalised state - in this case, $D$ is the standard MValgebra $[0,1]$ and the rest follows from Lemma 1. Moreover, by regarding Boolean algebras as algebras of the same similarity type as MV-algebras, we can consider the case when the function $s: E \rightarrow D$ is a homomorphism. Then Lemma 1 shows that $s$ is a generalised state. Moreover, in this case the range $s[E]$ is in fact a Boolean algebra, because $E$ is and $s$ is a homomorphism that is onto its range. Hence, $s[E]$ must be contained in the Boolean skeleton $B(D)$ of $D$ [4, Corollary 1.5.4], and $s[E]$ must be a Boolean subalgebra of $B(D)$. We thus have the following. Any homomorphism $s: E \rightarrow D$ is a generalised state that factors as in the commutative diagram below into generalised states which are surjective and injective, respectively, and a factorisation of $s$ with these properties is essentially unique:

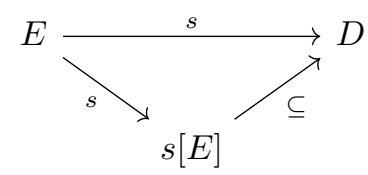

3.1 Example 1: The infinite lottery.

Let us model an experiment consisting in the random draw of a natural number $n \in \mathbb{N}$. Assume that the events to be considered ought to afford an answer at least to questions of the form ' $n \in A$ ?', for $A \subseteq \mathbb{N}$ finite. The minimal algebraic model of observable events is then the finite-cofinite Boolean algebra $\mathscr{E}$ on $\mathbb{N}$, that is,

$\mathscr{E}=\left\{A \subseteq \mathbb{N} \mid\right.$ either $A$ or $A^{\prime}$ is finite $\}$

Note that there is no $[0,1]$-valued state modelling both (i) the uniformly random character of the experiment, and (ii) the intuition that each $n \in \mathbb{N}$ may actually turn out to be the winning number. That is to say, there is no state $s: \mathscr{E} \rightarrow[0,1]$ satisfying $p(\{n\})=\alpha>0$ for all $n \in \mathbb{N}$. There is, however, exactly one state $s: \mathscr{E} \rightarrow[0,1]$ modelling (i) alone, namely, the invariant state given by

$s(A)=\left\{\begin{array}{ll}0 & A \text { finite, } \\ 1 & A \text { cofinite, }\end{array} \quad A \in \mathscr{E}\right.$.

This state $s$ fails to account for (ii), in that according to $s$ the probability that any given single $n \in \mathbb{N}$ be drawn is 0 . The classical way out of this situation is to issue the usual caveat that events of null probability may actually obtain - probability zero and impossibility are distinct concepts. Whether one considers this solution satisfactory or not, we proceed to exhibit an alternative way out that makes use of generalised states.

We construct a generalised state for the experiment at hand that accounts for both (i) and (ii). We do so by replacing the co-domain MV-algebra $[0,1]$ with 
a non-semisimple MV-chain, the well-known Chang's $M V$-algebra $C[3]$. Its underlying set is

$C=\{0, \varepsilon, 2 \varepsilon, \ldots, 1-2 \varepsilon, 1-\varepsilon, 1\}$

where $\varepsilon$ is a symbol interpreted as a positive infinitesimal and $C$ is equipped with the operations:

$x \oplus y= \begin{cases}(k+m) \varepsilon & x=k \varepsilon, y=m \varepsilon, \\ 1-(m-k) \varepsilon & x=k \varepsilon, y=1-m \varepsilon, m>k, \\ 1-(k-m) \varepsilon & x=1-k \varepsilon, y=m \varepsilon, k>m, \\ 1 & \text { otherwise, }\end{cases}$

$\neg x= \begin{cases}1-k \varepsilon & \text { if } x=k \varepsilon, \\ k \varepsilon & \text { if } x=1-k \varepsilon .\end{cases}$

We define a mapping $s: \mathscr{E} \rightarrow C$ such that, for all $A \in \mathscr{E}$,

$s(A)= \begin{cases}|A| \varepsilon & A \text { finite } \\ 1-\left|A^{\prime}\right| \varepsilon & A \text { cofinite }\end{cases}$

Lemma 1 can be employed to show that $s$ is a generalised state. First,

$s(\mathbb{N})=1-\left|\mathbb{N}^{\prime}\right| \varepsilon=1-0 \varepsilon=1$.

Second, take $A, B \in \mathscr{E}$ such that $A \cap B=\emptyset$. This implies that at least one of the sets must be finite. If $A$ and $B$ are finite, then

$s(A) \oplus s(B)=|A| \varepsilon \oplus|B| \varepsilon=|A \cup B| \varepsilon=s(A \cup B)$

and, analogously, $s(A) \odot s(B)=0$. Assume that $A$ is finite and $B$ is cofinite. Then the assumption $A \cap B=\emptyset$ yields $A \subseteq B^{\prime}$ and

$$
\begin{aligned}
s(A) \oplus s(B) & =|A| \varepsilon \oplus\left(1-\left|B^{\prime}\right| \varepsilon\right)=1-\left(\left|B^{\prime}\right|-|A|\right) \varepsilon \\
& =1-\left|A^{\prime} \cap B^{\prime}\right| \varepsilon=1-\left|(A \cup B)^{\prime}\right| \varepsilon \\
& =s(A \cup B),
\end{aligned}
$$

as $A \cup B$ is cofinite. Finally,

$s(A) \odot s(B)=|A| \varepsilon \odot\left(1-\left|B^{\prime}\right| \varepsilon\right)=\neg(\underbrace{(1-|A| \varepsilon) \oplus\left|B^{\prime}\right| \varepsilon}_{1})$,

since $|A| \leq\left|B^{\prime}\right|$. Hence, $s$ is a generalised state $\mathscr{E} \rightarrow C$.

The state $s: \mathscr{E} \rightarrow C$ of this example can be viewed as a non-standard analogue of the probabilistic model for the random selection of a natural number: there is a "uniform nowhere-zero distribution" on the sample space $\mathscr{E}$ intuitively associated to $s$, which is the function constantly equal to $\varepsilon$ on $\mathscr{E}$.
3.2 Example 2: Affine representations are generalised states.

In this example we apply the classical theory of affine representations of unital Riesz spaces, or more generally of unital lattice-ordered Abelian groups. See [8] for background. We formulate everything in the language of MV-algebras; this requires a straightforward translation from the group-theoretic version via the functor $\Gamma$. The Boolean algebra $E$ comes with its associated state space. To explain this, let St $E$ be the set of all [0,1]valued states of $E$, and consider the set-theoretic product $[0,1]^{E}$ equipped with its product (Tychonoff) topology. Using the natural (injective) map St $E \longrightarrow[0,1]^{E}$, we topologise St $E$ through the subspace topology induced by $[0,1]^{E}$. The resulting Tychonoff space St $E$ is the state space of $E$. It is well known that $\mathrm{St} E$ is a compact convex subset of the ambient locally convex topological linear space $\mathbb{R}^{E}$.

For any $a \in E$, let us define a function

$\hat{a}: \mathrm{St} E \longrightarrow[0,1]$

by setting, for every $p \in \operatorname{St} E$,

$\hat{a}(p):=p(a)$.

Then $\hat{a}$ is continuous and affine. To represent $E$ by such affine functions, let $\mathrm{C}(\mathrm{St} E)$ be the MV-algebra of all continuous functions St $E \rightarrow[0,1]$, with operations defined pointwise from those of the standard MV-algebra $[0,1]$. Define a function $s: E \rightarrow \mathrm{C}(\mathrm{St} E)$ as $s(a)=\hat{a}$, for every $a \in E$. Then $s$ provides such a representation. The reader is cautioned that $s$ is not a homomorphism, in general. However, it can be shown that $s$ indeed is a generalised state. Thus, the theory of affine representations of Boolean algebras may be recast in the language of generalised states.

We shall see at the end of our note that this example is a crucially important one: states arising from affine representations enjoy the universal property of free objects, cfr. Remark 4.

\section{State algebras}

In the Birkhoff-Lipson approach to multi-sorted ${ }^{1}$ algebras [2], classical universal algebra is extended to multisorted operations. The key to doing this is to replace the category of sets and functions by the category of multisorted sets and multi-sorted functions. Here we only

1 Called heterogeneous algebras in [2]. We stick to the multisorted terminology which seems to have become standard. 
spell out the two-sorted case of interest to us. A twosorted set is an ordered pair $(A, B)$ of sets. The component sets $A$ and $B$ are called the sorts of the two-sorted set $(A, B)$. A two-sorted function

$f:\left(A_{1}, B_{1}\right) \longrightarrow\left(A_{2}, B_{2}\right)$

between two-sorted sets is a pair $f:=\left(f_{1}, f_{2}\right)$ of functions

$f_{1}: A_{1} \longrightarrow A_{2}$,

$f_{2}: B_{1} \longrightarrow B_{2}$.

Composition and identity morphisms are defined component-wise. This defines the category of two-sorted sets. One can study multi-sorted sets equipped with multi-sorted operations in much the same way as one studies, in general algebra, sets equipped with operations. We shall apply this idea to generalised states.

Consider, then, a generalised state $s: E \rightarrow D$. We can regard the pair of sets $(E, D)$ as a two-sorted set; we call $E$ the sort of events, and $D$ the sort of probability degrees. Then $s: E \rightarrow D$ is a unary operation between the two different sorts. Hence, we are concerned with two-sorted algebras whose underlying two-sorted set is the ordered pair $(E, D)$, and whose (multi-sorted) operations are as follows.

(T1) The operations of Boolean algebras $\cup, \cap,{ }^{\prime}, \top, \perp$ in the single sort $E$.

(T2) The operations of MV-algebras $\oplus, \neg, 0$ in the single sort $D$.

(T3) The unary operation $s$ with sort $E$ in the domain and $D$ in the codomain.

Thus (T1-T3) define a two-sorted similarity type. Building on Definition 1, we now have:

Definition 2 (State algebra) A state algebra is an algebra $(E, D, s)$ of the two-sorted similarity type (T1T3) such that the following equational conditions hold.

(S1) $\left(E, \cup, \cap,^{\prime}, \perp, \top\right)$ is a Boolean algebra.

(S2) $(D, \oplus, \neg, 0)$ is an MV-algebra.

(S3) The operation $s: E \rightarrow D$ is a generalised state, i.e. satisfies the equational conditions (A1-A3) in Definition 1 .

When no danger of confusion arises, we write only $(E, D)$ in place of $(E, D, s)$.

All of the universal-algebraic constructions and fundamental theorems go through to the multi-sorted setting up to minimal modifications. ${ }^{2}$ For example, since state

\footnotetext{
2 Somewhat more substantial changes are needed in the presence of algebras with underlying multi-sorted sets which are not everywhere non-empty. In our case, given that the type of MV-algebras, hence of Boolean algebras, includes constants, neither $E$ nor $D$ can be empty.
}

algebras are defined by equations, they are closed under homomorphic images, subalgebras, and products. For an important sample of such universal-algebraic multisorted results please see the original [2]. We now focus on free objects.

For clarity, let us first spell out the notion of homomorphism of state algebras. A homomorphism between state algebras $\left(E_{1}, D_{1}\right)$ and $\left(E_{2}, D_{2}\right)$ is a morphism

$h:\left(E_{1}, D_{1}\right) \longrightarrow\left(E_{2}, D_{2}\right)$

of two-sorted sets, which means that $h:=\left(h_{1}, h_{2}\right)$ is a pair of functions $h_{1}: E_{1} \rightarrow E_{2}, h_{2}: D_{1} \rightarrow D_{2}$, such that $h$ preserves all operations in the type (T1-T3). In turn, the latter means that $h_{1}$ is a homomorphism of Boolean algebras, $h_{2}$ is a homomorphism of MValgebras, and $h$ preserves the operation $s$ - that is, the following diagram commutes:

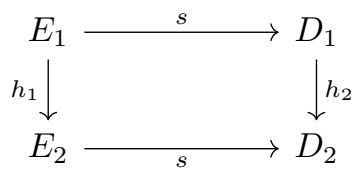

Elementwise, for all $a \in E_{1}$ we have

$h_{2}(s(a))=s\left(h_{1}(a)\right)$.

As in the classical, single-sorted case, free algebras always exist in equationally defined classes of multi-sorted algebras. We now state the defining universal property of free state algebras, which is obtained from the usual one for single-sorted algebras by replacing the category of sets with the category of two-sorted sets.

Definition 3 (Free state algebras) Let $\iota:\left(S_{1}, S_{2}\right) \rightarrow$ $(E, D)$ be a two-sorted function, where $\left(S_{1}, S_{2}\right)$ is a twosorted set, and $(E, D)$ is (the underlying two-sorted set of) a state algebra. We say $\iota$ is free (with respect to the class of all state-algebras) if for every two-sorted function $\eta:\left(S_{1}, S_{2}\right) \rightarrow\left(E^{\prime}, D^{\prime}\right)$, where $\left(E^{\prime}, D^{\prime}\right)$ is (the underlying two-sorted set of) a state algebra, there is exactly one homomorphism of state algebras

$h:(E, D) \longrightarrow\left(E^{\prime}, D^{\prime}\right)$

that makes the following diagram commute.

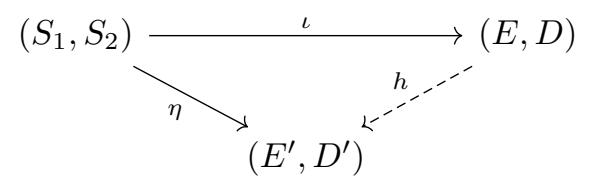

In standard algebraic usage, one refers to $(E, D)$ in Definition 3 as an algebra "freely generated by the two-sorted set" $\left(S_{1}, S_{2}\right)$, omitting reference to $\iota$. It can indeed be shown that $\left(\iota_{1}\left[S_{1}\right], \iota_{2}\left[S_{2}\right]\right)$, where $\iota:=\left(\iota_{1}, \iota_{2}\right)$, does generate $(E, D)$. However, care is needed with the notion 
of generating system in the multi-sorted case. We will see, for instance, that even when $\left(\iota_{1}\left[S_{1}\right], \iota_{2}\left[S_{2}\right]\right)$ freely generates $(E, D), \iota_{2}\left[S_{2}\right]$ need not generate - let alone freely generate - the MV-algebra $D$. This is in fact a general phenomenon about subalgebras in the twosorted setting, and has nothing to do with freeness; we illustrate it by the simplest possible example.

Example 1 Let $F_{1}$ be the Boolean algebra freely generated by $x_{1} \in F_{1}$, and consider the generalised state $s: F_{1} \rightarrow F_{1}$ given by the identity homomorphism. Then $\left(F_{1}, F_{1}\right)$ is a state algebra. The two-sorted subset $\left(\left\{x_{1}\right\}\right.$, $\left.\left\{x_{1}\right\}\right)$ obviously generates $\left(F_{1}, F_{1}\right)$, because $x_{1}$ generates the Boolean algebra $F_{1}$. Note, however, that also the two-sorted subset $\left(\left\{x_{1}\right\}, \emptyset\right)$ generates $\left(F_{1}, F_{1}\right)$. Indeed, apply first the Boolean operations in the sort of events to obtain $F_{1}$ from $x_{1}$. Then apply the operation $s$ to $x_{1}$, obtaining an element $s\left(x_{1}\right) \in F_{1}$ in the sort of probability degrees. Since $s$ is the identity, $s\left(x_{1}\right)=x_{1} \in F_{1}$; therefore, applying the MV-algebraic operations in the sort of probability degrees to $x_{1}$ yields the whole $F_{1}$.

It is easy to describe the state-algebras $(E, D)$ freely generated by a two-sorted set $\left(\emptyset, S_{2}\right)$, for $S_{2}$ an arbitrary set. Indeed, let $F M V\left(S_{2}\right)$ denote the MV-algebra freely generated by $S_{2}$, and let $\mathbf{2}$ be the two-element Boolean algebra, and consider the state algebra $\left(\mathbf{2}, F M V\left(S_{2}\right)\right)$ whose operation $s: \mathbf{2} \rightarrow F M V\left(S_{2}\right)$ is given by the only possible choice $-\mathbf{2}$ is the initial object in the category of MV-algebras. We leave it to the reader to verify that $\left(\mathbf{2}, F M V\left(S_{2}\right)\right)$ is the state algebra freely generated by $\left(\emptyset, S_{2}\right)$.

\section{Representation theorem for free state algebras}

In this section we prove our main result. For each integer $n \geq 0$, we let $F_{n}$ denote the Boolean algebra freely generated by the generators $x_{1}, \ldots, x_{n}$. Thus, $F_{0}=\mathbf{2}$, the two-element Boolean algebra. We shall obtain a representation theorem for the state algebras freely generated by a multi-sorted set $\left(\left\{x_{1}, \ldots, x_{n}\right\}, \emptyset\right)$ in terms of $F_{n}$ and an appropriate generalised state of $F_{n}$. The state algebra freely generated by $(\emptyset, \emptyset)$ is immediately seen to be $(\mathbf{2}, \mathbf{2})$ with $s: \mathbf{2} \rightarrow \mathbf{2}$ the only possible such generalised state, namely, the identity. Thus we assume $n \geq 1$ throughout.

In order to formulate and prove our result concisely, we will assume familiarity with basic polyhedral geometry, McNaughton functions, the geometric representation theory of finitely generated free MV-algebras, and the MV-algebraic theory of Schauder hats. The needed background can be found in [16].
In the Euclidean $n$-dimensional linear space $\mathbb{R}^{n}$, let $e_{1}, \ldots, e_{n}$ denote the standard orthonormal basis, let $[0,1]^{n} \subseteq \mathbb{R}^{n}$ denote the unit $n$-cube, and let $\Delta_{n}:=$ conv $\left\{e_{1}, \ldots, e_{n}\right\}$ denote the standard $(n-1)$-simplex, the convex hull of the standard basis. Let us further write $\pi_{i}:[0,1]^{n} \rightarrow[0,1]$ for the projection functions, $i=1, \ldots, n$, and let $\mathrm{C}\left([0,1]^{n}\right)$ be the MV-algebra of all continuous $[0,1]$-valued functions on the unit $n$-cube. (Cfr. Section 3.2.) By $\mathscr{M}_{n}$ we denote the MV-subalgebra of $\mathrm{C}\left([0,1]^{n}\right)$ generated by $\left\{\pi_{i}\right\}_{i=1}^{n}$. Then it is known that $\mathscr{M}_{n}$ is in fact freely generated by $\left\{\pi_{i}\right\}_{i=1}^{n}$, and consists precisely of the McNaughton functions, that is, the continuous piecewise-linear functions whose affine linear pieces have integer coefficients. For any subset $X \subseteq[0,1]^{n}$ we write

$$
\mathscr{M}_{n}(X)
$$

for the MV-algebra obtained by restricting the elements of $\mathscr{M}_{n}$ to $X$. Writing

$r: \mathscr{M}_{n} \longrightarrow \mathscr{M}_{n}(X)$

for the restriction map, $r$ is a surjective MV-homomorphism whose kernel ker $r:=\left\{f \in \mathscr{M}_{n} \mid r(f)=0\right\}$ is the ideal of all McNaughton functions vanishing over $X$, or equivalently (by continuity), over the closure of $X$. We specifically consider the MV-algebra

$\mathscr{S}_{n}:=\mathscr{M}_{n}\left(\Delta_{n}\right)$

which we call the $M V$-algebra dual to the standard $n$ simplex. It will be useful to have an explicit description of the kernel of

$\rho: \mathscr{M}_{n} \longrightarrow \mathscr{S}_{n}$.

We already used the standard fact that, in any MValgebra $M$ with enveloping lattice-ordered Abelian group with unit $(G, 1)$, and for any $x, y \in M$, the equality $x \oplus y=x+y$ holds if, and only if, $x \odot y=0$. This shows that the condition $x+y=1$ is equationally expressible in $\mathrm{MV}$-algebraic language by the system of equations

$\left\{\begin{array}{l}x \oplus y=1 \\ x \odot y=0 .\end{array}\right.$

It is an MV-algebraic exercise to extend this from two to $n$ summands. For an explicit description of the MValgebraic relations ${ }^{3}$ needed, please see [15]. What matters for us is that the partition of unity condition

$x_{1}+\cdots+x_{n}=1$,

3 Since universal algebraists reserve the name 'equation' for fully invariant identities, in the following we use 'relation' to mean an equality between two MV-algebraic terms that holds for given elements of a given MV-algebra. 
for $x_{1}, \ldots, x_{n} \in M$, while a priori only meaningful in $(G, 1)$, is in fact expressible in the MV-algebra $M$ by finitely many equivalent relations in the variables $x_{1}, \ldots, x_{n}$. Since it is elementary that any finite set of $\mathrm{MV}$-algebraic relations is equivalent to a single one of the form $\tau=0$, for $\tau$ an appropriate MV-algebraic term, we shall write $\sigma\left(x_{1}, \ldots, x_{n}\right)$ for a term in the variables $x_{1}, \ldots, x_{n}$ that satisfies

$\sigma\left(x_{1}, \ldots, x_{n}\right)=0$

in $M$ if, and only if, $(*)$ holds in $(G, 1)$.

We can now consider the principal ideal

$I_{n}:=\left\langle\sigma\left(\pi_{1}, \ldots, \pi_{n}\right)\right\rangle$

of $\mathscr{M}_{n}$ generated by the element $\sigma\left(\pi_{1}, \ldots, \pi_{n}\right) \in \mathscr{M}_{n}$, along with the quotient MV-algebra

$\frac{\mathscr{M}_{n}}{I_{n}}$.

Our discussion above of the definition of $I_{n}$ shows that the zero set of $I_{n}$ - the set of points in $[0,1]^{n}$ where the functions in $I_{n}$ jointly vanish — is precisely the standard simplex $\Delta_{n} \subseteq[0,1]^{n}$. It follows immediately (by the contravariant Galois correspondence between zero sets and ideals) that the restriction map $\rho$ in $(\dagger)$ factors through the natural quotient map $\mathscr{M}_{n} \rightarrow \frac{\mathscr{M}_{n}}{I_{n}}$. But since $I_{n}$ is principal, an application of the HayWójcicki Theorem yields that the second component of this factorisation - i.e., the comparison map between quotient objects - is in fact an isomorphism $\frac{\mathscr{M}_{n}}{I_{n}} \rightarrow$ $\mathscr{S}_{n}$. Thus, in conclusion,

$\operatorname{ker} \rho=I_{n}$,

which provides the promised explicit description of the kernel of $\rho$.

Next, let us consider $F_{n}$, the Boolean algebra freely generated by the set $\left\{x_{1}, \ldots, x_{n}\right\}$. Let us display the $2^{n}$ atoms of $F_{n}$ as

$m_{1}, \ldots, m_{2^{n}}$

the usual convention is that $m_{j}$ is the unique atom of $F_{n}$ such that the binary expansion of $j-1$ has 1 in its $i^{\text {th }}$ digit if, and only if, $m_{j}$ lies below the generator $x_{i}$ in the underlying order of $F_{n}$. To each $m_{j}$ there corresponds a uniquely determined vertex of the standard simplex $\Delta_{2^{n}}$, namely, the unit vector $e_{j}$.

Lemma 2 Fix an integer $n \geq 1$. Each generalised state $s: F_{n} \rightarrow D$ is uniquely determined by its action on the set $\left\{m_{1}, \ldots, m_{2^{n}}\right\}$ of atoms of $F_{n}$.
Proof Indeed, by elementary Boolean algebra, for each $b \in F_{n}$ there is a unique $J_{b} \subseteq\left\{1, \ldots, 2^{n}\right\}$ such that

$b=\bigcup_{j \in J_{b}} m_{j}$.

Now $m_{j} \cap m_{k}=\perp$ whenever $j \neq k$. By Lemma 1 , using addition of the enveloping lattice-group of $D$, we have

$s(b)=\sum_{j \in J_{b}} s\left(m_{j}\right)$,

and the statement is proved.

We supplement the preceding lemma with a simple but crucial existence result:

Lemma 3 Fix an integer $n \geq 1$. Consider a function

$\bar{s}:\left\{m_{1}, \ldots, m_{2^{n}}\right\} \longrightarrow D$

from the set of atoms of the Boolean algebra $F_{n}$ freely generated by $\left\{x_{1}, \ldots, x_{n}\right\}$ to an $M V$-algebra $D$. The following are equivalent.

1. There is a unique extension of $\bar{s}$ to a generalised state $s: F_{n} \rightarrow D$.

2. In the (essentially unique) Abelian lattice-group $G$ with unit 1 such that $\Gamma(G, 1)=D$, we have

$$
\sum_{j=1}^{2^{n}} \bar{s}\left(m_{j}\right)=1 .
$$

Proof The implication from item 1 to item 2 is given by Lemma 1. For the converse implication, suppose item 2 holds. For each $b \in F_{n}$, set

$s(b):=\sum_{j \in J_{b}} s\left(m_{j}\right)$,

where $J_{b}$ is as in Lemma 2. Then the function $s: F_{n} \rightarrow$ $D$ extends $\bar{s}$ because $J_{m_{j}}=\left\{m_{j}\right\}$ for each $j=1, \ldots, 2^{n}$. Now, if $b_{1} \cap b_{2}=\perp$, it must be the case that $J_{b_{1}} \cap J_{b_{2}}=$ $\emptyset$, and then

$J_{b_{1} \cup b_{2}}=J_{b_{1}} \cup J_{b_{2}}$, 
where the union is disjoint. Therefore,

$$
\begin{aligned}
s\left(b_{1} \cup b_{2}\right) & =s\left(\bigcup_{j \in J_{b_{1}}} m_{j} \cup \bigcup_{j \in J_{b_{2}}} m_{j}\right) \\
& =s\left(\bigcup_{j \in\left(J_{b_{1}} \cup J_{b_{2}}\right)} m_{j}\right) \\
& \left.=s\left(\bigcup_{j \in J_{b_{1} \cup b_{2}}} m_{j}\right) \quad \text { (By def. of } s .\right) \\
& =\sum_{j \in J_{b_{1}} \cup b_{2}} s\left(m_{j}\right) \\
& =\sum_{j \in\left(J_{b_{1}} \cup J_{b_{2}}\right)} s\left(m_{j}\right) \\
& =\sum_{j \in J_{b_{1}}} s\left(m_{j}\right)+\sum_{j \in J_{b_{2}}} s\left(m_{j}\right) \\
& =s\left(b_{1}\right)+s\left(b_{2}\right),
\end{aligned}
$$

and $s$ is additive. By Lemma 1 , it remains to show that $s$ is normalised, i.e. satisfies $s(T)=1$. But since $\top=$ $\bigcup_{j=1}^{2^{n}} m_{j}$, this is a consequence of additivity together with the assumption in item 2 .

Remark 3 Although here we only need the case of finite free algebras, it is clear that the statement in Lemma 2 holds for any finite Boolean algebra, with the same proof - atoms determine finitely additive probabilities in atomic Boolean algebras. For an extension of this basic fact to the (atomless!) free finitely generated MValgebras of many-valued events, see [13, Lemma 7.2]. Lemma 3 also allows for other generalisations which we do not pursue here.

Next, to each vertex $e_{j}$ of $\Delta_{2^{n}}$ there corresponds the Schauder hat $H_{j}$ — the pyramidal function — with apex at $e_{j}$ which is affine linear on each face of $\Delta_{2^{n}}$. Of course, $\left\{H_{j}\right\}_{j=1}^{2^{n}} \subseteq \mathscr{S}_{2^{n}}$. Also, it is elementary that

$\sum_{j=1}^{2^{n}} H_{j}=1$,

where we use ' 1 ' to denote the function constantly equal to 1 over $\mathscr{S}_{2^{n}}$.

Lemma 4 There exists exactly one generalised state $s: F_{n} \rightarrow \mathscr{S}_{2^{n}}$ that satisfies

$s\left(m_{j}\right)=H_{j}$

for each $j=1, \ldots, 2^{n}$.

Proof There is at most one such state, by Lemma 2. In light of $(b)$ and Lemma 3 , there is at least one.
Definition 4 For an integer $n \geq 1$ we define the free state algebra to be the state algebra $\left(F_{n}, \mathscr{S}_{2^{n}}\right)$, where $F_{n}$ is the Boolean algebra freely generated by $n$ elements $x_{1}, \ldots, x_{n}, \mathscr{S}_{2^{n}}$ is the MV-algebra dual to the standard simplex $\Delta_{2^{n}}$, and $s: F_{n} \rightarrow \mathscr{S}_{2^{n}}$ is the unique generalised state in Lemma 4.

Theorem 1 For each integer $n \geq 1$, the free state algebra $\left(F_{n}, \mathscr{S}_{2^{n}}\right)$ is freely generated by its multi-sorted subset $\left(\left\{x_{1}, \ldots, x_{n}\right\}, \emptyset\right)$.

Proof Write $\iota:\left(\left\{x_{1}, \ldots, x_{n}\right\}, \emptyset\right) \rightarrow\left(F_{n}, \mathscr{S}_{2^{n}}\right)$ for the two-sorted inclusion function $\iota:=\left(\iota_{1}, \iota_{2}\right)$ such that $\iota_{1}$ is the inclusion map $\left\{x_{1}, \ldots, x_{n}\right\} \subseteq F_{n}$, and $\iota_{2}: \emptyset \rightarrow \mathscr{S}_{2^{n}}$ is the only possible function. Let $\left(E^{\prime}, D^{\prime}\right)$ be any state algebra, and consider an arbitrary function

$\eta_{1}:\left\{x_{1}, \ldots, x_{n}\right\} \longrightarrow E^{\prime}$,

which yields the obvious two-sorted function

$\eta:\left(\left\{x_{1}, \ldots, x_{n}\right\}, \emptyset\right) \longrightarrow\left(E^{\prime}, D^{\prime}\right)$.

We define a function

$\bar{h}_{1}:\left\{x_{1}, \ldots, x_{n}\right\} \longrightarrow E^{\prime}$

by setting

$\bar{h}_{1}\left(x_{i}\right):=\eta_{1}\left(x_{i}\right), i=1, \ldots, n$.

Since $F_{n}$ is freely generated by $\left\{x_{i}\right\}_{i=1}^{n}$, there is exactly one homomorphism

$h_{1}: F_{n} \longrightarrow E^{\prime}$

that extends $\bar{h}_{1}$. We define a second function

$\bar{h}_{2}:\left\{s\left(m_{1}\right), \ldots, s\left(m_{2^{n}}\right)\right\} \longrightarrow D^{\prime}$

by setting

$\bar{h}_{2}\left(s\left(m_{j}\right)\right):=s\left(h_{1}\left(m_{j}\right)\right), j=1, \ldots, 2^{n}$.

(For the sake of clarity, let us stress that the symbol ' $s$ ' on the right-hand side of (D) denotes the generalised state of $\left(D^{\prime}, E^{\prime}\right)$, whereas the one on the left-hand side is the generalised state in Lemma 4.) We show that $\bar{h}_{2}$, too, has a unique extension to a homomorphism. By (D) and Lemma 4 we have

$\bar{h}_{2}\left(H_{j}\right)=s\left(h_{1}\left(m_{j}\right)\right), j=1, \ldots, 2^{n}$.

Further, let us prove

$\sum_{j=1}^{2^{n}} s\left(h_{1}\left(m_{j}\right)\right)=1$.

Indeed, $h_{1}$ is a Boolean homomorphism and $\left\{m_{j}\right\}_{j=1}^{2^{n}}$ is a pairwise disjoint set whose join is $T$, which implies 
that $\left\{h_{1}\left(m_{j}\right)\right\}_{j=1}^{2^{n}} \subseteq E^{\prime}$ is a pairwise disjoint set that joins to $T$; by the additivity of $s$,

$\sum_{j=1}^{2^{n}} s\left(h_{1}\left(m_{j}\right)\right)=s\left(\bigcup_{j=1}^{2^{n}} h_{1}\left(m_{j}\right)\right)=1$,

as was to be shown. Now, by the equality $(\sharp)$ and the universal property of quotients, the set $\left\{H_{j}\right\}_{j=1}^{2^{n}}$ generates the MV-algebra $\mathscr{S}_{2^{n}}$ freely ${ }^{4}$ subject to the relation (b). Since that relation was just shown to hold for $\left\{s\left(h_{1}\left(m_{j}\right)\right)\right\}_{i=1}^{2^{n}}$, there is a unique extension of $\bar{h}_{2}$ to a homomorphism

$h_{2}: D \longrightarrow D^{\prime}$

Setting

$h:=\left(h_{1}, h_{2}\right):\left(F_{n}, \mathscr{S}_{2^{n}}\right) \longrightarrow\left(E^{\prime}, D^{\prime}\right)$

yields one homomorphism of state algebras that satisfies the universal property of free state algebras. The proof of the fact that any such homomorphism coincides with $h$ is a straightforward direct verification, and is left to the reader.

Remark 4 The reader can now check the following. Let $s^{\prime}: E \rightarrow \mathrm{C}(\mathrm{St} E)$ be the affine representation of the Boolean algebra $E$, as defined in Example 2 of Section 3. Let $D \subseteq \mathrm{C}(\mathrm{St} E)$ be the MV-subalgebra of $\mathrm{C}(\mathrm{St} E)$ generated by the image $s^{\prime}[E]$ of $E$ under $s^{\prime}$. Then we have a factorisation

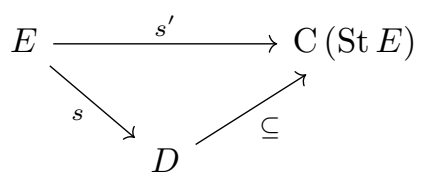

where $s: E \rightarrow D$ is a generalised state which yields a state algebra $(E, D)$. Set $E:=F_{n}$ in the foregoing, for $n \geq 1$ an integer. Then, as a corollary of Theorem 1: The state algebra $(E, D)$ is freely generated by the multi-sorted set $\left(\left\{x_{1}, \ldots, x_{n}\right\}, \emptyset\right)$. Thus free state algebras arise from affine representations of Boolean algebras.

\section{Further research}

We briefly comment on a few research directions related to the algebraic framework for states presented here.

4 The theory of the relations satisfied by Schauder bases, which leads to the notion of abstract Schauder bases, was developed in the two papers [11], [12]. We see here a simple instance of that theory in action.
- Fuzzy Probability Logic FP(Ł) over infinite-valued Łukasiewicz logic was developed by Hájek in $[10$, Chapter 8.4]. The purpose of $\mathrm{FP}(\mathrm{E})$ is to formalize reasoning about properties of states. The distinctive feature of $\mathrm{FP}(\mathrm{E})$ is its two-level syntax: probability assessments are syntactically represented in the language by a unary modality read 'Probably', which applies to Boolean formulas only. The class of Boolean algebras and states provides a possible complete semantics for $\mathrm{FP}(\mathrm{E})$. It would be desirable, however, to develop an equivalent algebraic semantics in the varietal sense. State algebras seem to provide such an equivalent (multi-sorted) algebraic semantics for $\mathrm{FP}(\mathrm{E})$. We plan to give an account of this in a further paper.

- The traditional use of MV-algebras is to model degrees of truth in Eukasiewicz logic. In this note, however, MV-algebras are employed as abstract models of degrees of probability - for the first time, as far as we know. Like Janus, MV-algebras show one of their two faces according as they are used by way of domain or co-domain of a generalised state. This phenomenon calls for further reflection and research.

- One can apply Robinson's non-standard analysis in order to introduce infinitesimal degrees of probability. This requires fairly heavy machinery from first-order logic and model theory. In our Example 1, by contrast, equational algebra only suffices: MV-algebraic infinitesimals are considerably simpler than Robinson's infinitesimals. One is thus able to formally develop a theory of "infinitesimal degrees of probability" using standard algebra only. We plan to elaborate on this point in subsequent investigation.

\section{References}

1. A. Bigard, K. Keimel, and S. Wolfenstein. Groupes et anneaux réticulés, Lecture Notes in Mathematics, Vol. 608. Springer-Verlag, Berlin-New York, 1977.

2. G. Birkhoff and J. D. Lipson. Heterogeneous algebras. J. Combinatorial Theory, 8:115-133, 1970.

3. C. C. Chang. Algebraic analysis of many valued logics. Transactions of the AMS, 88:467-490, 1958.

4. R. L. O. Cignoli, I. M. L. D'Ottaviano, and D. Mundici. Algebraic foundations of many-valued reasoning, volume 7 of Trends in Logic-Studia Logica Library. Kluwer Academic Publishers, Dordrecht, 2000.

5. L. C. Ciungu, G. Georgescu, and C. Mureşan. Generalized Bosbach states: Part II. Archive for Mathematical Logic, 52(7-8):707-732, 2013.

6. T. Flaminio and T. Kroupa. States of MV-algebras. In P. Cintula, C. Fermuller, and C. Noguera, editors, Handbook of Mathematical Fuzzy Logic. Volume 3, Studies in Logic, Mathematical Logic and Foundations. College Publications, London, 2015. 
7. T. Flaminio and F. Montagna. MV-algebras with internal states and probabilistic fuzzy logics. International Journal of Approximate Reasoning, 50(1):138-152, 2009.

8. K. R. Goodearl. Partially ordered abelian groups with interpolation, volume 20 of Mathematical Surveys and Monographs. American Mathematical Society, Providence, RI, 1986.

9. T. Hailperin. Sentential probability logic: Origins, development, current status, and technical applications. Lehigh University Press, 1996.

10. P. Hájek. Metamathematics of fuzzy logic, volume 4 of Trends in Logic-Studia Logica Library. Kluwer Academic Publishers, Dordrecht, 1998.

11. C. Manara, V. Marra and D. Mundici. Lattice-ordered abelian groups and Schauder bases of unimodular fans Trans. Amer. Math. Soc., 359(4):1593-1604, 2007.

12. V. Marra. Lattice-ordered abelian groups and Schauder bases of unimodular fans, II. Trans. Amer. Math. Soc., 365(5):2545-2568, 2013.

13. V. Marra and D. Mundici. The Lebesgue state of a unital abelian lattice-ordered group. J. Group Theory, 10(5):655684, 2007.

14. D. Mundici. Averaging the truth-value in Łukasiewicz logic. Studia Logica, 55(1):113-127, 1995.

15. D. Mundici. Reasoning on imprecisely defined functions. In Discovering the world with fuzzy logic, volume 57 of Stud. Fuzziness Soft Comput., pp. 331-366. Physica, Heidelberg, 2000.

16. D. Mundici. Advanced Lukasiewicz calculus and $M V$ algebras, volume 35 of Trends in Logic-Studia Logica Library. Springer, Dordrecht, 2011.

17. S. Pulmannová. A note on observables on MV-algebras. Soft Computing, 4(1):45-48, 2000. 